\title{
Some Approximate Schemes for Solving Nonlinear Equations
}

\author{
Muqadssa Shahzadi \\ Department of Mathematics, COMSATS University Islamabad, Islamabad, Pakistan \\ e-mail: muqadssa1995@gmail.com
}

\begin{abstract}
Some iterative algorithms for solving nonlinear equation $f(x)=0$ are suggested and investigated using Taylor series and homotopy perturbation technique. These algorithms can be viewed as extensions and generalization of well known methods such as Householder and Halley methods with cubic convergence. Convergence of the proposed methods has been discussed and analyzed. Several numerical examples are given to illustrate the efficiency of the suggested algorithms for solving nonlinear equations. Comparison with other iterative schemes is carried out to show the validity and performance of these algorithms.
\end{abstract}

\section{Introduction}

Solution of nonlinear equations are an important area of research in numerical analysis. In this branch of mathematics "Numerical analysis" usually deal with the continuous problem which come through daily life. These problems of real world phenomena occur in mathematics and all fields of sciences, such as, biology, economics, and physics.

We were familiar about the technique of hand calculation before the use of computers. Before the use of numerical algorithms analog devices were been used extensively in the science and amongst all the scientific Rule was common to the engineers which develop by Gunter in 1620 .

Received: January 30, 2022; Accepted: February 15, 2022

2020 Mathematics Subject Classification: 65K99.

Keywords and phrases: homotopy perturbation method, nonlinear equations, Halley methods, Householder method, Newtons method. 
A nonlinear method in mathematics and science in which performance shift is not proportional to the input transition. Engineers, biologists, mathematicians and several other scientific scientists are involved in nonlinear problem. Newton method play central role in the development of technique for optimization most of current physical method for optimization can be viewed as variant form of the Newton method.

In many cases, it is not always possible to find the exact solution of nonlinear equations. Thus we use the approximate methods to find the solutions of nonlinear equations. For finding iterative approaches are usually applied for the solution of the nonlinear equation $f(x)=0$. For instance the method of bisection, the Taylor series and the method of homotopy perturbation method. The homotopy perturbation method is used to solve a problem in pure and applied mathematics in different fields of science. This method is quiet efficient and allow us for choosing the auxiliary parameter arbitrary. The perturbation technique is used to solve the nonlinear problems. However the technique of perturbation based on the assumption of small parameters. Most of the nonlinear equation involving small parameter does not exist. These facts motivated the researchers to find approximate solutions of nonlinear equations $f(x)=0$. These methods are as follows:

(i) Bisection method

(ii) Newton method

(iii) Regula Falsi method

(iv) Fixed point Iterative method

(v) Secant method

In recent years, many modification have been established to above mention methods for computing nonlinear equations $f(x)=0$. These methods have been constructed using different techniques such as Taylor series. Numerical methods have many application in pure and applied sciences. Most of the problems in applied and pure mathematics may lead to solve the nonlinear equations. For 
more details, see [1, 2, 3, 4, 5, 6, 7, 8, 9, 10, 11, 12, 13, 14, 15, 16, 17, 18, 19, 20, 21, 22, 23, 24] and the reference therein. All the numerical methods which we used in this work to solve nonlinear equation have their own imitation and drawbacks. Thus its essential to discuss the efficiency of numerical methods but also to aware about its disadvantages. The working rule of numerical methods is to find the approximate solutions of any mathematical problems on a defined interval either in time or distance. After the hard work has been done by ancient Egyptian the Newton's and Leibniz developed calculus which led the foundation to solve the mathematical problems.

Inspired and motivated by the research activities in this dynamic and fascinating fields, we use the homotopy perturbation technique, which was introduced and studied by He [8, 9]. Noor [16, 17, 18, 19, 20, 21] and Noor et al. 22. modified the homotopy perturbation technique to suggest a wide class of iterative methods for solving nonlinear equations and related optimization problems. Some new iterative methods are proposed for solving nonlinear equations. To illustrate the efficiency of the methods, some numerical examples are given. Comparions withe other known methods shows that the new methods perform better than the previous known methods.

\section{Main Results}

The homotopy perturbation method was introduced by He, [8, 9] it has been used in many branches of mathematics for finding solutions. Abbasbandy [1] applied the homotopy perturbation technique to derive various iterative methods for solving nonlinear equations. We again use this technique to suggest and propose some new iterative methods for solving the nonlinear equations. To be more precise, we consider the problem of solving the type of nonlinear equation of the type

$$
f(x)=0,
$$


which can be decomposed as:

$$
L(x)=N(x)+c,
$$

where $L$ is a linear operator, $N$ is a non-linear operator and $c$ is an arbitrary constant. Using the technique of [ [] , we can define homotopy $H(v, p): R \times[0,1] \rightarrow$ $R$ as:

$$
\begin{aligned}
H(v, p) & =(1-p)\left[L(v)-L\left(v_{0}\right)\right]+p[L(v)-N(v)-c] \\
& =L(v)-p N(v)-c=0, \quad p \in[0,1] .
\end{aligned}
$$

Note that for $p=0, p=1$ and from equation (3) we have,

$$
\begin{aligned}
& H(v, 0)=L(v)-L\left(v_{0}\right)=v-c=0 \\
& H(v, 1)=[L(v)-N(v)]-c=0 .
\end{aligned}
$$

Here $p \in[0,1]$ is the embedding parameter which is increasing monotonically from 0 to 1 . This implies that $H(v, 0)=L(v)-c=v-c=0$ is continuously deformed to basic problem as: $H(v, 1)=L(v)-N(v)-c=v-N(v)-c=0$.

Let the solution of (1) can be expressed as

$$
v=v_{0}+p v_{1}+p^{2} v_{2}+\ldots
$$

The approximate solution of the nonlinear equation (1) is obtained as:

$$
V=\lim _{p \rightarrow 1} v=v=v_{0}+v_{1}+v_{2}+\ldots .
$$

We can write equation (3) by using (5) and expanding Taylor series $N(v)$ around $v_{0}$ as

$$
\begin{aligned}
v_{0}+p v_{1}+p^{2} v_{2}+\ldots & =p^{0} c+p\left[N\left(v_{0}\right)+\left(v-v_{0}\right) N^{\prime}\left(v_{0}\right)+\ldots\right] \\
& =p^{0} c+p\left[N\left(v_{0}\right)+p\left(v_{1}+p v_{2}+\cdots\right) N^{\prime}\left(v_{0}\right)+\ldots\right] \\
& \left.=p^{0} c+p N\left(v_{0}\right)\right)+p^{2} v_{1} N^{\prime}\left(v_{0}\right) .
\end{aligned}
$$


By comparing the coefficient of $p$, we have

$$
\begin{array}{ll}
p^{0} & : \quad v_{0}=c \\
p^{1} & : \quad v_{1}=N\left(v_{0}\right) \\
p^{2} & : \quad v_{2}=v_{1} N^{\prime}\left(v_{0}\right)
\end{array}
$$

Consequently, we have

$$
v_{0}+v_{1}+v_{2}+\ldots=c+N\left(v_{0}\right)+v_{1} N^{\prime}(0)+\ldots .
$$

We use the equation (9) to obtain the main algorithm for the solution of nonlinear equation (1). First of all, we consider the equations

$$
e^{\alpha x} f(x)=0, \quad \alpha \in R .
$$

Using the Taylor series, we obtain

$$
\begin{array}{r}
0=e^{\alpha \gamma} f(x)=e^{\alpha \gamma} f(\gamma)+(x-\gamma) e^{\alpha \gamma}\left[f^{\prime}(\gamma)+\alpha f(\gamma)\right] \\
+\frac{1}{2 !}(x-\gamma)^{2} e^{\alpha \gamma}\left[f^{\prime \prime}(\gamma)+2 \alpha\left(f^{\prime}(\gamma)\right)+\alpha^{2} f(\gamma)\right],
\end{array}
$$

which implies that

$0=f(\gamma)+(x-\gamma)\left[f^{\prime}(\gamma)+\alpha f^{\prime}(\gamma)\right]+\frac{1}{2 !}(x-\gamma)^{2}\left[f^{\prime \prime}(\gamma)+2 \alpha\left(f^{\prime}(\gamma)+\alpha^{2} f(\gamma)\right)\right]+\ldots$

Solving equation 12 for $x$, we have

$$
\begin{aligned}
x & =\gamma-\frac{f(\gamma)}{f^{\prime}(\gamma)+\alpha f(\gamma)}-\frac{1}{2 !}(x-\gamma)^{2} \frac{\left[f^{\prime \prime}(\gamma)+2 \alpha f^{\prime}(\gamma)+\alpha^{2} f(\gamma)\right]}{\left[f^{\prime}(\gamma)+\alpha f(\gamma)\right]} \\
& =c+N(x),
\end{aligned}
$$

where

$$
\begin{aligned}
c & =\gamma-\frac{f(\gamma)}{\left[f^{\prime}(\gamma)+\alpha f(\gamma)\right]} \\
N(x) & =-\frac{1}{2 !}(x-\gamma)^{2} \frac{\left[f^{\prime \prime}(\gamma)+2 \alpha f^{\prime}(\gamma)+\alpha^{2} f(\gamma)\right]}{\left[f^{\prime}(\gamma)+\alpha f(\gamma)\right]}
\end{aligned}
$$


Also

$$
N^{\prime}(x)=-(x-\gamma) \frac{\left[f^{\prime \prime}(\gamma)+2 \alpha f^{\prime}(\gamma)+\alpha^{2} f(\gamma)\right]}{\left[f^{\prime}(\gamma)+\alpha f(\gamma)\right]} .
$$

From (9) and (14), we have

$$
\begin{aligned}
& x_{0}=c=\gamma-\frac{f(\gamma)}{\left[f^{\prime}(\gamma)+\alpha f(\gamma)\right]}, \\
& x_{1}=N\left(v_{0}\right)=-\frac{1}{2 !}\left(x_{0}-\gamma\right)^{2} \frac{\left[f^{\prime \prime}(\gamma)+2 \alpha f^{\prime}(\gamma)+\alpha^{2} f(\gamma)\right]}{\left[f^{\prime}(\gamma)+\alpha f(\gamma)\right]} \\
& x_{2}=v_{1} N^{\prime}\left(v_{0}\right)=v_{1}\left\{-\left(x_{0}-\gamma\right) \frac{\left[f^{\prime \prime}(\gamma)+2 \alpha f^{\prime}(\gamma)+\alpha^{2} f(\gamma)\right]}{\left[f^{\prime}(\gamma)+\alpha f(\gamma)\right]}\right\}
\end{aligned}
$$

From (17), it follows that approximate $x \approx x_{0}$ such that

$$
x=c=\gamma-\frac{f(\gamma)}{\left[f^{\prime}(\gamma)+\alpha f(\gamma)\right]}
$$

which help us to suggest the following iterative scheme.

Algorithm I: For a given initial value $x_{0}$, compute the approx value $x_{n}+1$ by the iterative scheme:

$$
x_{n+1}=x_{n}-\frac{f\left(x_{n}\right)}{\left[f^{\prime}\left(x_{n}\right)+\alpha f\left(x_{n}\right)\right]} \quad n=0,1,2,3, \ldots,
$$

which is well known modified Newton's method and has quadratic convergence.

From (17) and (18), we obtain

$$
x_{1}=-\frac{1}{2 !}\left[\frac{f(\gamma)}{f^{\prime}(\gamma)+\alpha f(\gamma)}\right]^{2} \frac{\left[f^{\prime \prime}(\gamma)+2 \alpha f^{\prime}(\gamma)+\alpha^{2} f(\gamma)\right]}{\left[f^{\prime}(\gamma)+\alpha f(\gamma)\right]} .
$$

One can find the approximate $x$ as

$$
\begin{aligned}
x & \approx x_{0}+x_{1}=x_{0}+N\left(x_{0}\right) \\
& =\gamma-\frac{f(\gamma)}{f^{\prime}(\gamma)+\alpha f(\gamma)}-\frac{1}{2 !}\left[\frac{f(\gamma)}{f^{\prime}(\gamma)+\alpha f(\gamma)}\right]^{2} \frac{\left[f^{\prime \prime}(\gamma)+2 \alpha f^{\prime}(\gamma)+\alpha^{2} f(\gamma)\right]}{\left[f^{\prime}(\gamma)+\alpha f(\gamma)\right]} .
\end{aligned}
$$


This enable us to suggest the following one step method.

Algorithm II: For a given $x_{0}$, compute the approx sol $x_{n+1}$ by iterative scheme

$$
\begin{aligned}
x_{n+1} & =x_{n}-\frac{f\left(x_{n}\right)}{\left[f^{\prime}\left(x_{n}\right)+\alpha f\left(x_{n}\right)\right]} \\
& -\frac{1}{2 !}\left[\frac{f\left(x_{n}\right)}{f^{\prime}\left(x_{n}\right)+\alpha f\left(x_{n}\right)}\right]^{2}\left[\frac{f^{\prime \prime}\left(x_{n}\right)+2 \alpha f^{\prime}\left(x_{n}\right)+\alpha^{2} f\left(x_{n}\right)}{f^{\prime}\left(x_{n}\right)+\alpha f\left(x_{n}\right)}\right], \quad n=0,1,2, \ldots
\end{aligned}
$$

From (18), 19) and (21), we have,

$$
\begin{aligned}
x_{2} & =x_{1} N^{\prime}\left(x_{0}\right)=N\left(x_{0}\right) N^{\prime}\left(x_{0}\right) \\
& =\frac{1}{2 !}\left(x_{0}-\gamma\right)^{3}\left(\frac{f^{\prime \prime}(\gamma)+2 \alpha f^{\prime}(\gamma)+\alpha^{2} f(\gamma)}{f^{\prime}(\gamma)+\alpha f(\gamma)}\right)^{2} .
\end{aligned}
$$

Consequently, we have

$$
\begin{aligned}
x \approx & x_{0}+x_{1}+x_{2}=x_{0}+N\left(x_{0}\right)+x_{1} N^{\prime}(0) \\
= & \gamma-\frac{f(\gamma)}{\left[f^{\prime}(\gamma)+\alpha f(\gamma)\right]}-\frac{1}{2 !}\left(x_{0}-\gamma\right)^{2} \frac{\left[f^{\prime \prime}(\gamma)+2 \alpha f^{\prime}(\gamma)+\alpha^{2} f(\gamma)\right]}{\left[f^{\prime}(\gamma)+\alpha f(\gamma)\right]}+ \\
& \frac{1}{2 !}\left(x_{0}-\gamma\right)^{3}\left[\frac{f^{\prime \prime}(\gamma)+2 \alpha f^{\prime}(\gamma)+\alpha^{2} f(\gamma)}{f^{\prime}(\gamma)+\alpha f(\gamma)}\right]^{2} .
\end{aligned}
$$

This formulation help us to construct a following algorithms.

Algorithm III: The following iterative method is used to obtain $x_{n+1}$ as $x_{0}$ is provided

$$
\begin{aligned}
x_{n+1}= & x_{n}-\frac{f\left(x_{n}\right)}{\left[f^{\prime}\left(x_{n}\right)+\alpha f\left(x_{n}\right)\right]}-\frac{1}{2 !}\left(x_{n+1}-\gamma\right)^{2} \frac{\left[f^{\prime \prime}\left(x_{n}\right)+2 \alpha f^{\prime}\left(x_{n}\right)+\alpha^{2} f\left(x_{n}\right)\right]}{\left[f^{\prime}\left(x_{n}\right)+\alpha f\left(x_{n}\right)\right]} \\
& +\frac{1}{2 !}\left(x_{n+1}-\gamma\right)^{3}\left[\frac{f^{\prime \prime}\left(x_{n}\right)+2 \alpha f^{\prime}\left(x_{n}\right)+\alpha^{2} f\left(x_{n}\right)}{f^{\prime}\left(x_{n}\right)+\alpha f\left(x_{n}\right)}\right]^{2}
\end{aligned}
$$

which is an implicit method.

To implement the implicit method, we use the predictor-corrector technique. Using modified Newton method as predictor and Algorithm III as corrector method, we can suggest the following two-step method. 
Algorithm IV: For a given initial value $x_{0}$, compute the approximate value $x_{n+1}$ by the iterative scheme

$$
\begin{aligned}
y_{n}= & x_{n}-\frac{f\left(x_{n}\right.}{f^{\prime}\left(x_{n}\right)+\alpha f\left(x_{n}\right)} \\
x_{n+1}= & y_{n}-\frac{1}{2 !}(x-\gamma)^{2}\left(\frac{f^{\prime \prime}\left(x_{n}\right)+2 \alpha f^{\prime}\left(x_{n}\right)+\alpha^{2} f\left(x_{n}\right)}{f^{\prime}\left(x_{n}\right)+\alpha f\left(x_{n}\right)}\right) \\
& +\frac{1}{2 !}\left(y_{n}-\gamma\right)^{3}\left(\frac{f^{\prime \prime}\left(x_{n}\right)+2 \alpha f^{\prime}\left(x_{n}\right)+\alpha^{2} f\left(x_{n}\right)}{f^{\prime}\left(x_{n}\right)+\alpha f\left(x_{n}\right)}\right)^{2},
\end{aligned}
$$

which is equivalent to the following method.

Algorithm V: For a given initial value $x_{0}$, compute the approximate solution $x_{n+1}$ by the iterative scheme:

$$
\begin{aligned}
x_{n+1}= & x_{n}-\frac{f\left(x_{n}\right)}{f^{\prime}\left(x_{n}\right)+\alpha f\left(x_{n}\right)} \\
& -\frac{1}{2 !}\left(\frac{f\left(x_{n}\right)}{f^{\prime}\left(x_{n}\right)+\alpha f\left(x_{n}\right)}\right)^{2}\left(\frac{f^{\prime \prime}\left(x_{n}\right)+2 \alpha f^{\prime}\left(x_{n}\right)+\alpha^{2} f\left(x_{n}\right)}{f^{\prime}\left(x_{n}\right)+\alpha f\left(x_{n}\right)}\right) \\
& -\frac{1}{2 !}\left(\frac{f\left(x_{n}\right)}{f^{\prime}\left(x_{n}\right)+\alpha f\left(x_{n}\right)}\right)^{3}\left(\frac{f^{\prime \prime}\left(x_{n}\right)+2 \alpha f^{\prime}\left(x_{n}\right)+\alpha^{2} f\left(x_{n}\right)}{f^{\prime}\left(x_{n}\right)+\alpha f\left(x_{n}\right)}\right)^{2},
\end{aligned}
$$

which is an exlipcit method and appears to be new one.

\section{Numerical Illustration}

In this section, we check some numerical examples to illustrate the efficiency of our suggested new developed algorithm for solving nonlinear equation. Numerical result are compared with other well known method like Newton method (NM), Householder method (HM) and Halley method. The stopping criteria followed by $\left|x_{n+1}-x_{n}\right|<10^{-15}$.

Example 1: Consider the nonlinear equation $f(x)=x^{2}-e^{x}-3 x+2=0$, $x_{0}=0.5$. 
Table 1: Comparison of NM, HM with Algorithm II and Algorithm III.

\begin{tabular}{|c|c|c|c|c|c|}
\hline \multicolumn{3}{|l|}{ Method } & $\mathbf{N}$ & $v x_{n}$ & $f\left(x_{n}\right)$ \\
\hline \multicolumn{3}{|l|}{ NM } & 5 & 0.257530285439861 & 0 \\
\hline \multicolumn{3}{|l|}{ HM } & 4 & 0.257530285439861 & 0 \\
\hline \multicolumn{3}{|l|}{ HHM } & 4 & 0.257530285439861 & 0 \\
\hline \multirow[t]{4}{*}{$4^{*}$ Algorithm ll } & \multirow[t]{4}{*}{$4^{*} \alpha$} & -1 & 5 & 0.257530285439861 & 0 \\
\hline & & -0.5 & 4 & 0.257530285439861 & 0 \\
\hline & & -0.25 & 4 & 0.257530285439861 & 0 \\
\hline & & 0 & 4 & 0.257530285439861 & 0 \\
\hline \multirow[t]{4}{*}{$4^{*}$ Algorithm 111} & \multirow[t]{4}{*}{$4^{*} \alpha$} & -1 & 4 & 0.257530285439861 & 0 \\
\hline & & -0.5 & 4 & 0.257530285439861 & $4.440892098500626 \mathrm{e}^{\wedge}-016$ \\
\hline & & -0.25 & 4 & 0.257530285439861 & 0 \\
\hline & & 0 & 4 & 0.257530285439861 & 0 \\
\hline
\end{tabular}

Example 2: Consider $f(x)=e^{x^{2}+7 x-30}-1, x_{0}=3.5$.

Table 2: Comparision of NM, HH, with algorithm III and Algorithm IV.

\begin{tabular}{|c|c|c|c|c|c|}
\hline \multicolumn{3}{|l|}{ Method } & Iteration & $f_{n}$ & $f\left(x_{n}\right)$ \\
\hline \multicolumn{3}{|l|}{ NM } & 13 & 3 & $3.289812866569264 \mathrm{e}-012$ \\
\hline \multicolumn{3}{|l|}{ HM } & 7 & 3 & $7.105427357601002 \mathrm{e}-015$ \\
\hline \multicolumn{3}{|l|}{ HHM } & 9 & 3 & 0 \\
\hline \multirow[t]{4}{*}{$4 *$ Algorithm 111} & \multirow[t]{4}{*}{$4^{*} \alpha$} & -1 & 8 & 3 & $1.421085471520200 \mathrm{e}-014$ \\
\hline & & -0.5 & 8 & 3 & $8.935074902183260 \mathrm{e}-012$ \\
\hline & & -0.25 & 8 & 3 & $1.444497854663496 \mathrm{e}-010$ \\
\hline & & 0 & 9 & 3 & 0 \\
\hline \multirow[t]{4}{*}{ 4*Algorithm lV } & \multirow[t]{4}{*}{$4^{*} \alpha$} & -1 & 7 & 3 & 0 \\
\hline & & -0.5 & 7 & 3 & $4.440892098500626 \mathrm{e}^{\wedge}-016$ \\
\hline & & -0.25 & 7 & 3 & 0 \\
\hline & & 0 & 7 & 3 & $7.105427357601002 \mathrm{e}-015$ \\
\hline
\end{tabular}


Example 3: $\quad$ Consider $f(x)=\sin ^{2} x-x^{2}+1, x_{0}=1$.

Table 3: Comparison of NM, HM, with new Alogrithm III.

\begin{tabular}{|c|c|c|c|c|c|}
\hline \multicolumn{3}{|l|}{ Method } & Iteration & $x_{n}$ & $f\left(x_{n}\right)$ \\
\hline \multicolumn{3}{|l|}{ NM } & 6 & 1.404491648215341 & $-7.593925488436071 \mathrm{e}-013$ \\
\hline \multicolumn{3}{|l|}{ HM } & 4 & 1.404491648215341 & $6.667999485898690 \mathrm{e}-013$ \\
\hline \multicolumn{3}{|l|}{ HHM } & 6 & 1.404491648215341 & $-4.440892098500626 \mathrm{e}-016$ \\
\hline \multirow[t]{4}{*}{$4^{*}$ Algorithm 111} & \multirow[t]{4}{*}{$4^{*} \alpha$} & -1 & 4 & 1.404491648215341 & $-4.440892098500626 \mathrm{e}-016$ \\
\hline & & -0.5 & 4 & 1.404491648215341 & $2.764455331316640 \mathrm{e}-014$ \\
\hline & & -0.25 & 5 & 1.404491648215341 & $3.330669073875470 \mathrm{e}-016$ \\
\hline & & 0 & 6 & 1.404491648215341 & $-4.440892098500626 \mathrm{e}-016$ \\
\hline \multirow[t]{4}{*}{$4^{*}$ Algorithm lV } & \multirow[t]{4}{*}{$4^{*} \alpha$} & -1 & 4 & 1.404491648215341 & $-4.440892098500626 \mathrm{e}-016$ \\
\hline & & -0.5 & 4 & 1.404491648215341 & $3.330669073875470 \mathrm{e}-016$ \\
\hline & & -0.25 & 4 & 1.404491648215341 & $1.221245327087672 \mathrm{e}-015$ \\
\hline & & 0 & 4 & 1.404491648215341 & 6.667999485898690e-013 \\
\hline
\end{tabular}

Table 3 shows that new Algorithms are more efficient than Newton method.

Example 4: Consider $f(x)=x^{3}-2 x^{2}+2, x_{0}=0$.

Table 4: Comparison of NM, HM with Algorithms III.

\begin{tabular}{|c|c|c|c|c|c|}
\hline \multicolumn{3}{|l|}{ Method } & Iteration & $x_{n}$ & $f\left(x_{n}\right)$ \\
\hline \multicolumn{3}{|l|}{ NM } & - & fails & - \\
\hline \multicolumn{3}{|l|}{ HM } & - & fails & - \\
\hline \multicolumn{3}{|l|}{ HHM } & - & fails & - \\
\hline \multirow[t]{5}{*}{$5 *$ Algorithm 111} & \multirow[t]{5}{*}{$5^{*} \alpha$} & 1 & 5 & -0.83928675521416 & 0 \\
\hline & & 0 & fail & - & - \\
\hline & & -0.25 & 22 & -0.839286755214161 & 0 \\
\hline & & -0.5 & 10 & -0.839286755214161 & $2.22044604925031 \mathrm{e}-16$ \\
\hline & & -1 & 18 & -0.839286755214161 & $2.22044604925031 \mathrm{e}-16$ \\
\hline \multirow[t]{5}{*}{$5^{*}$ Algorithm ll } & \multirow[t]{5}{*}{$5^{*} \alpha$} & 1 & 5 & -0.8392286755214161 & 0 \\
\hline & & 0.5 & 5 & -0.839286755214161 & 0 \\
\hline & & 0.25 & 6 & -0.839286755214161 & 0 \\
\hline & & 0 & fail & - & - \\
\hline & & -0.5 & -0.5 & -0.839286755214161 & $2.220446049250313 \mathrm{e}-16$ \\
\hline
\end{tabular}


These numerical results indicate that the suggested algorithms perform better then the Newton method and have the same defines as Householder and Halley method. In the last example shows that the newly developed algorithms give approximate solution of given nonlinear equation, where Newton, Householder and Halley method fail to find approximate.

\section{References}

[1] S. Abbasbandy, Improving Newton-Raphson method for nonlinear equation by modified decomposition method, App. Math. Comput. 145 (2003), 887-893. https://doi.org/10.1016/S0096-3003(03)00282-0

[2] K. E. Atkinson, An Introduction to Numerical Analysis, John Wiley and Sons, New York, USA, 1987.

[3] E. Bobolian and J. Biazar, Solution of nonlinear equation by modified Adomain decomposition method, Appl. Math. Comput. 132 (2002), 167-172.

https://doi.org/10.1016/S0096-3003(01)00184-9

[4] R. L. Burden and J. D. Faires, Numerical Analysis, PWS Publishing Company, Boston, USA, 2001.

[5] C. Chun, and Y. Ham, Some fourth-order modifications of Newton method, Applied Mathematics and Computation 197(2) (2008), 654-658. https://doi.org/10.1016/j.amc.2007.08.003

[6] A. Cordero, C. Jordan and J. R. Torregrosa, One point Newton type iterative method: A unified point of view, Appl. Math. Comput. 275 (2015), 366-374. https://doi.org/10.1016/j.cam.2014.07.009

[7] R. Escobedo, and L. L. Bonilla, Numerical method for quantum drift diffusion equation in semiconductor physics, J. Math. Chem. 40(1) (2006), 3-13. https://doi.org/10.1007/s10910-006-9122-9

[8] J. H. He, Homotopy perturbation technique, Comp. Math. Appl. Mech. Eng. 178 (1999), 257-262. https://doi.org/10.1016/S0045-7825(99)00018-3

[9] J. H. He, A new iterative method for solving algebraic equations, Appl. Math. Comp. 135 (2005), 81-84. https://doi.org/10.1016/S0096-3003(01)00313-7 
[10] A. S. Householder, The Numerical Treatment of a Single Nonlinear Equations, McGraw-Hill, New York, 1970.

[11] T. Lotfi, S. Shateyi and S. Hadadi, Potra-Ptak iterative method with memory, International Scholarly Research Notices 2014 (2014), Art. ID 697642, 6 pp. https://doi.org/10.1155/2014/697642

[12] X. Luo, A note on the new iteration for solving algebraic equations, Appl. Math. Comput. 171 (2005), 1177-1183. https://doi.org/10.1016/j.amc.2005.01.124

[13] M. A. Noor and K. I. Noor, Some iterative schemes for nonlinear equations, Appl. Math. Comput. 183 (2006), 774-779. https://doi.org/10.1016/j.amc.2006.05. 084

[14] K. I. Noor, M. A. Noor and S. Momani, Modified Householder iterative method for nonlinear equation, Appl. Math. Comput. 190 (2007), 1534-1539.

https://doi.org/10.1016/j.amc.2007.02.036

[15] K. I. Noor and M. A. Noor, Predictor-corrector Halley method for nonlinear equations, Appl. Math. Comput. 188 (2007), 1578-1591.

https://doi.org/10.1016/j.amc.2006.11.023

[16] M. A. Noor, New family of iterative methods for nonlinear equations, Appl. Math. Comput. 190 (2007), 553-558. https://doi.org/10.1016/j.amc.2007.01.045

[17] M. A. Noor, Homotopy perturbation method for solving nonlinear equation, J. Math. Anal. Approx. Theory 2 (2007), 111-117.

[18] M. A. Noor, New iterative schemes for nonlinear equitation, Appl. Math. Comput. 187 (2007), 937-943. https://doi.org/10.1016/j.amc.2006.09.028

[19] M. A. Noor, Some iterative methods for nonlinear equation using homotopy perturbation method, Int. J. Comput. Math. 87 (2010), 141-149.

https://doi.org/10.1080/00207160801969513

[20] M. A. Noor, Iterative method for nonlinear equation using homotopy perturbation method, Appl. Math. Inform. Sci. 4 (2010), 227-2235.

[21] M. A. Noor, Numerical Analysis and Optimization, Lecture Notes, Mathematics Department, COMSATS University Islamabad, Islamabad, Pakistan, 2008-2021. 
[22] M. A. Noor and W. A. Khan, New iterative method for solving nonlinear by using homotopy perturbation method, Appl. Math. Comput. 219 (2012), 3565-3574. https://doi.org/10.1016/j.amc.2012.09.040

[23] M. A. Noor and F. A. Shah, Higher order iterative scheme for nonlinear equation using decomposition technique, Appl. Math. Comput. 266 (2015), 414-423. https://doi.org/10.1016/j.amc.2015.05.054

[24] M. A. Waseem, M. A. Noor, K. I. Noor and F. A. Shah, An efficient technique to solve nonlinear equations using multiplication calculus, Turk. J. Math. 42 (2018), 679-691. https://doi.org/10.3906/mat-1611-95

This is an open access article distributed under the terms of the Creative Commons Attribution License (http://creativecommons.org/licenses/by/4.0/), which permits unrestricted, use, distribution and reproduction in any medium, or format for any purpose, even commercially provided the work is properly cited. 